\title{
Narrow-band filter for satellite communication systems
}

\author{
Alexander Vladimirovich Strizhachenko ${ }^{1}$, Sergey Nikolayevich Shulga ${ }^{2}$ \\ ${ }^{1}$ Department of Biophysics and Information Technologies, National University of Pharmacy, Kharkiv, Ukraine \\ ${ }^{2}$ Department of Theorenical Radiophysics, V. N. Karazin Kharkiv National University, Kharkiv, Ukraine
}

\section{Article Info}

Article history:

Received May 10, 2021

Revised Oct 27, 2021

Accepted Nov 10, 2021

\section{Keywords:}

Filter passband

Microwave

Satellite communications

Single crystal

Waveguide resonator

\begin{abstract}
Design narrow-band compact filters, based on high-quality waveguidedielectric resonators with anisotropic materials is the subject of this paper. Filter represents a segment of a rectangular waveguide rotated around the longitudinal axis of the waveguide 90 degrees and containing one or more dielectric inserts that completely fill the resonator along the narrow wall of the waveguide and partially along the wide one. A distinctive feature of the proposed filter is higher slope steepness of the amplitude-frequency characteristic, and high manufacturability in the centimeter range. The designed narrow-band filter satisfies contradictory requirements: it combines narrow bandwidth $\left(\approx 0.1 \%\right.$ of center frequency $\left.f_{0}\right)$ with low passband insertion loss $(\leq 1 \mathrm{~dB})$.
\end{abstract}

This is an open access article under the CC BY-SA license.

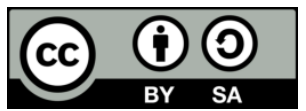

Corresponding Author:

Alexander Vladimirovich Strizhachenko,

Department of Biophysics and Information Technologies, National University of Pharmacy

53, Pushkinskaya Str., Kharkiv, Ukraine

Email: a.strizhachenko@gmail.com

\section{INTRODUCTION}

Microwave filters for various purposes are widely used in radio and telecommunications, radar and radio navigation systems for military, aerospace and general use, measuring equipment [1]-[9]. Information transfer is carried out through telephone networks, computer data networks, satellite communication systems, cellular radio communication systems. The growth of information flows requires an increase in both the capacity of the channels and the speed of their transmission in the line. Fiber optic, infrared and microwave digital backbone data transmission channels have become widespread. Microwave channels are formed by centimeter and millimeter-wave radio-relay lines, which effectively service information flows in computer networks and mobile cellular networks. The technical characteristics of radio-relay stations are largely dependent on the electrical parameters of the used band-pass filters. For example, the number of stations required to overlap the span of a line depends on the insertion loss of filters, while the slope steepness of their amplitude-frequency characteristics will determine the noise immunity and packing density of channels on electromagnetic high-frequency carrier during reception and transmission of information.

The variety of existing frequency - separative microwave devices indicates the ambiguity of solving of tasks assigned to them. Comparing the known microwave filters with a minimum of losses (with the same bandwidth or barrier), then it is possible to note, the waveguide filters have the best intrinsic quality factor [10]-[15]. This is explained by the fact that in the centimeter wavelength range, the waveguide (WR) and waveguide-dielectric resonators (WDR) [16]-[19] have the greatest natural quality factor. Waveguide filters have proven themselves in stationary equipment, where the requirements of minimum signal loss are more important than mass and dimensional parameters. However, for small-sized electronic equipment (used, for example, television systems, satellite communications and in aviation), decisive factors are weight and size 
indicators. At the same time, the accumulated experience on the miniaturization of microwave filters allows us to conclude that the energy and mass-dimensional parameters are in conflict with each other. In the process of finding a compromise between these indicators, filters based on anisotropic dielectrics are proposed. The proposed filters are somewhat inferior in terms of mass and size parameters of microstrip filters [1], [2], [7], [10], but they have significantly lower initial bandwidth losses (the main drawback of microstrip filters is the low $Q$ of the resonator elements $(Q \approx 200-250)$, as a result of which narrow-band filters large initial losses in the passband). Waveguide-dielectric filters have a significantly higher quality factor $Q$. In addition, the waveguide-dielectric filters allow you to transmit high power (more than 100 watts in uninterrupted mode). A distinctive feature of the proposed filters is a high intrinsic quality factor $(Q>20,000)$, a rare spectrum of parasitic resonances, high the slope steepness of amplitude-frequency characteristics, and also high manufacturability in the centimeter range.

\section{RESEARCH METHOD}

The proposed high-quality waveguide-dielectric resonator is made on the basis of a leucosapphire insert in the ultraboundary waveguide for the wave $\mathrm{H}_{10}$. At the same time, this segment of a rectangular waveguide with a dielectric is not ultraboundary for some orthogonal oscillations. The design of such a system (Figures 1,2 and 3) is a segment of a standard waveguide with a cross section, but rotated around the longitudinal axis of the waveguide by 90 degrees (Figure 1). A monocrystalline insert (one or more) is installed in the center of this segment of a rectangular waveguide. Connection with the bringing waveguide duct is done by docking it with a segment of a rectangular waveguide so that their longitudinal axes coincide, and the wide walls of the waveguide segment with the dielectric are parallel to the narrow walls of the bringing waveguide duct.

By turning the resonator through 90 degree, relatively to the waveguide H-plane (Figure 1), the Qfactor can be significantly increased as compared with waveguide-dielectric resonators for which the Qfactor is 5,000 [10]-[13], [16], [17]. This is due to the fact that the electric field near the metal is "drawn" into the dielectric. That, in turn, makes it possible to reduce the average loss power in the metal walls of the resonator. Such a filter satisfies contradictory requirements: it combines narrow bandwidth $(\approx 0.1 \%$ of center frequency $f_{0}$ ) with low passband insertion loss $\alpha_{0}\left(\alpha_{0} \leq 1 \mathrm{~dB}\right)$. Figure 4 shows the amplitude-frequency characteristic (AFC) for the single-section filter (1) and for the two-section filter (2) (that were measured on a C4-60 spectrum analyzer). The two-section filter (Figure 3) differs from a single section filter (Figure 2) in that it has two similar dielectric samples and the length $L$ is $12 \mathrm{~mm}$ longer $(L=42 \mathrm{~mm})$ than the length of the single-section filter $(L=30 \mathrm{~mm})$. An important filter characteristic is the squareness coefficient $K_{\alpha d B}$ (or the slope steepness of amplitude-frequency characteristics) - the ratio of the bandwidth at the level $\alpha_{\text {stop }}$ to the bandwidth at the minimum loss level $\alpha_{0}$. As known the slope steepness of amplitude-frequency characteristic determines the noise immunity and packing density of channels during reception and transmission of information. The squareness coefficient of the developed two section filter is $K_{-40 d B}=1.85$ (Figure 4). This coefficient $K_{\alpha d B}$ corresponds to the squareness coefficient of a five section filter based on the traditional WDR [10], [11], [16]. This will reduce the mass and size of the waveguide-dielectric filters at least half. Also, the squareness coefficient of the developed two section filter is 1.3-1.8 times better than the squareness coefficient of microstrip filters with the same passband [1], [3], [7]. Besides the losses in the passband $\alpha_{0}$ for the microstrip filters are $-2.5-3 \mathrm{~dB}[7]$.

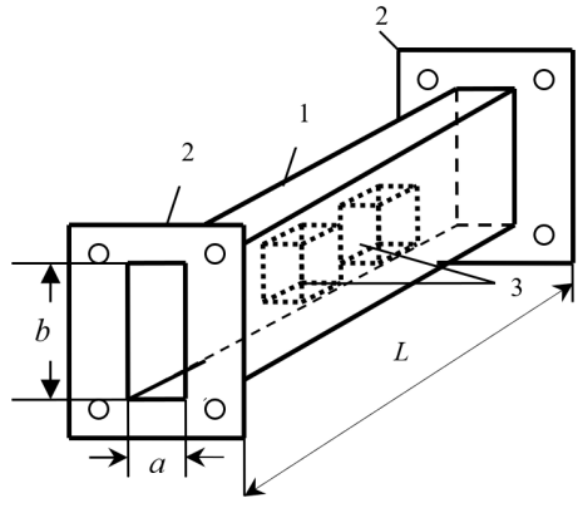

Figure 1. The design of a two-section filter

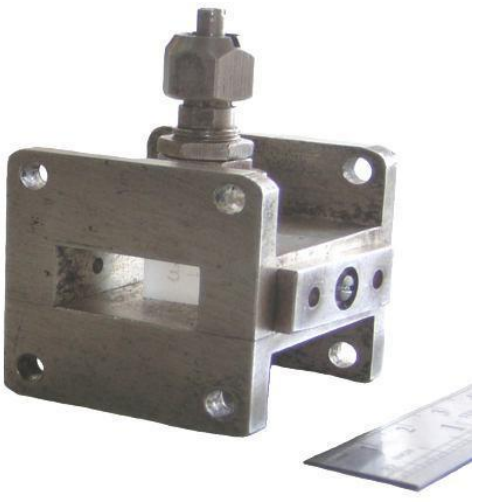

Figure 2. Single-section filter 


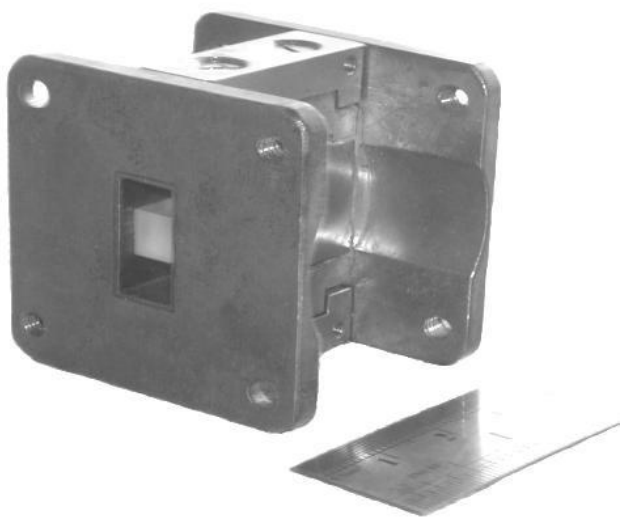

Figure 3. Two sections filter

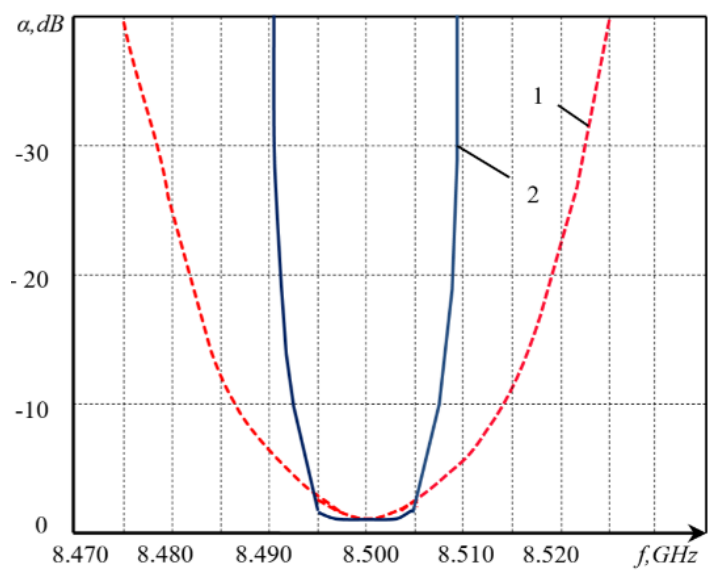

Figure 4. AFC for the single section filter

The main characteristics of the filters:

- $\quad$ Resonant frequency $\mathrm{fO}, \mathrm{MHz}$

Passband (on $1 \mathrm{~dB}$ level), $\mathrm{MHz}$

9.5

Initial losses $\alpha 0, \mathrm{~dB}$

$\approx 0.9$

Out-of-band suppression:

- for two-resonator filter, $\mathrm{dB}$

25

- for three- resonator filter, $\mathrm{dB}$

40

Frequency instability in the

- temperature range $-50-+650 \mathrm{C}, \mathrm{MHz}$

$\pm 2$

- Mass, g

- Dielectric

leucosapphire

\section{RESULTS AND DISCUSSION}

In this paper, the issue of the technological effectiveness in the mass production of such filters is investigated: influence of the state of the waveguide surfaces and the dielectric insert, the choice of construction material, as well as the tolerances and adjustment.

The influence of the material of the structure on the resonant wavelength $(\lambda)$ and it's intrinsic $\mathcal{Q}$ factor $\left(\mathcal{Q}_{0}\right)$. The comparison HQ WDRs based on the brass $\left(\sigma=1.57 \times 10^{7} \mathrm{~S} / \mathrm{m}\right)$ waveguide section and copper $\left(\sigma=5.97 \times 10^{7} \mathrm{~S} / \mathrm{m}\right)$ waveguide section has been performed. The results showed that the material of the waveguide section has a significant impact on the $\mathcal{Q}_{0}$-factor $\left(\mathcal{Q}_{0}=13500\right.$ for the brass sections; $\mathcal{Q}_{0}=19300$ for the copper sections) and practically does not affect the wavelength. Thus, the most suitable materials of waveguide sections are copper and silver.

To investigate the influence of the dielectric, insert material, three inserts with the same geometric dimensions have been produced. Inserts were made of leucosapphire $\left(\varepsilon=9.2-11.6, \operatorname{tg} \delta=5 \times 10^{-6}[20]\right)$, crystalline quartz $\left(\varepsilon=4.6, \operatorname{tg} \delta=5 \times 10^{-5}[20]\right)$ and fluoroplastic $\left(\varepsilon=2.2, \operatorname{tg} \delta=(2-3) \times 10^{-4}[20]\right)$. The results show a clear dependence of the intrinsic $\mathcal{Q}_{0}$-factor from the material of the dielectric insert $\left(\mathcal{Q}_{0}=5090\right.$ for fluoroplastic; $\mathcal{Q}_{0}=9740$ for crystalline quartz; $\mathcal{Q}_{0}=21600$ for leucosapphire). Therefore, it is necessary to select samples from crystalline materials.

The question of the tolerance influence on the resonator parameters in the process of manufacturing and assembling has been investigated as well. The main assembling of the filter was in the correct installation of the dielectric insert into the waveguide section. A symmetrical arrangement of the dielectric inserts with respect to the side walls of the waveguide and flanges is optimum for obtaining the highest performance of filter. The displacement $(\Delta L)$ of the insert relative to its symmetric position along the resonator length $(L)$ (relatively to the flanges) was carried out (Figure 5 and 6). The insert was shifted and controlled with a watch-type position micrometer with an accuracy of $0.1 \mathrm{~mm}$. The resonance frequency (curve 1 on Figure 5), the half-power bandwidth $(\Delta f)$ (curve 2, Figure 5), HQ resonator loss on a frequency of resonance (Figure 6, curve 1) and $\mathrm{Q}$ values of the resonator (curve 2, Figure 6) were monitored. It can be seen from the diagrams that displacement of the dielectric sample from the central position along the longitudinal axis of the 
resonator for a distance to $\pm 1 \mathrm{~mm}$ does not cause a noticeable change in the bandwidth of the resonator, resonant wavelength and attenuation on resonant wavelength.

The displacement of the dielectric inserts from the optimal position perpendicularly to the longitudinal axis of the resonator (along the dimension $b$ ) was investigated. The diagram in Figure 7 shows the dependence of the resonant wavelength $(a / \lambda)$ (curve 1) and the bandwidth (curve 2) on the displacement of the dielectric insert along the along the waveguide walls $(\Delta b)$. The diagram (Figure 8 ) represents the change $\alpha_{0}$ (curve 2), the loaded quality factor $\mathrm{Q}_{l}$ (curve 3) and the intrinsic quality factor $\mathrm{Q}_{0}$ (curve 1 ) of the resonator.

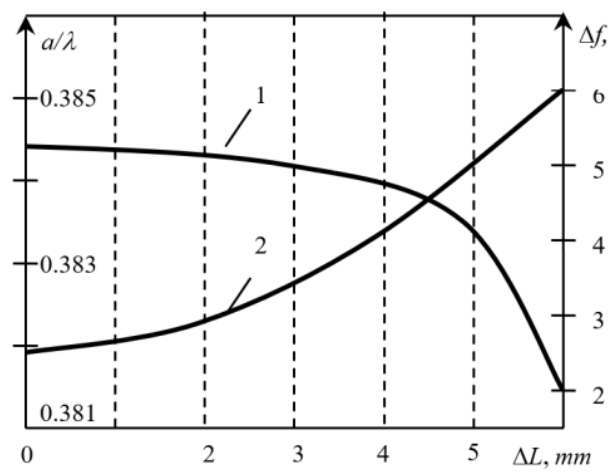

Figure 5. Dependence of the wavelength $a / \lambda$ and pass bandwidth $\Delta f$ on the displacement $\Delta L$ from the optimal position along the resonator length $L$

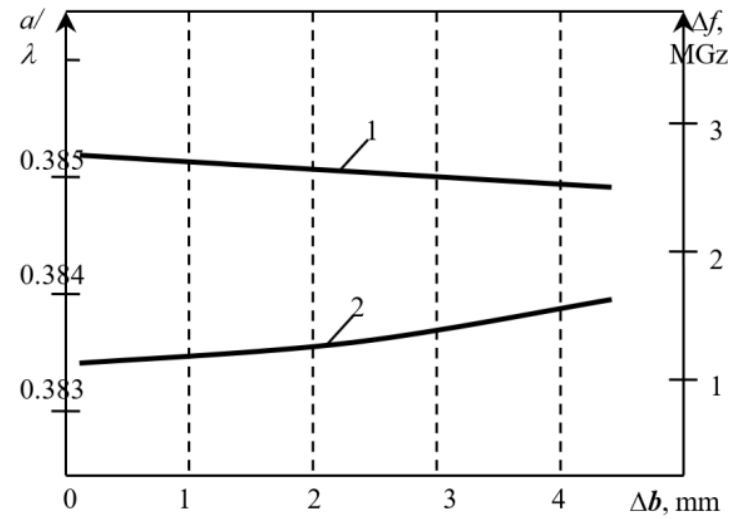

Figure 7. Dependence of the wavelength $a / \lambda$ (curve 1) and bandwidth $\Delta f$ (curve 2 ) on $\Delta b$ (along $b$ dimension)

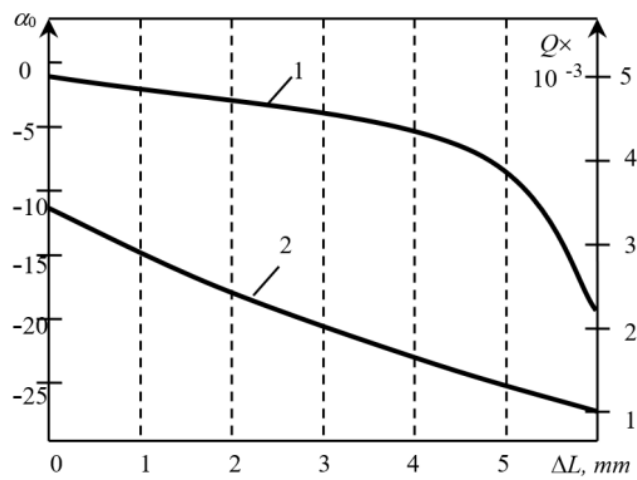

Figure 6. Dependence of the attenuation $\alpha_{0}$ and $Q$ values of the resonator on the displacement $\Delta L$ from the optimal position along the resonator length

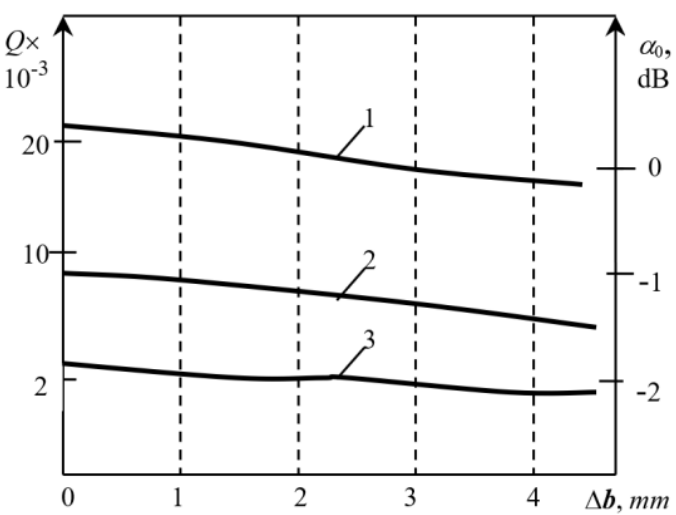

Figure 8 . The change $\alpha_{0}$ (curve 2), $Q_{0}$ (curve 1) and $Q_{l}$ (curve 3 ) from $\Delta b$

As can be seen, the displacement of the dielectric inserts from the symmetric position along the dimension $b$ to the value $\Delta b=4.5 \mathrm{~mm}$ has little effect on the resonance wavelength, bandwidth, and Q-factor. Further displacement leads to a increase of initial loss $\alpha_{0}$ (by about $0.35 \mathrm{~dB} / \mathrm{mm}$ ) and decrease of the resonator intrinsic $Q_{0}$ (by $3000 / \mathrm{mm}$ ). Hence it follows that the effect of displacement of the dielectric insert towards the side walls of the wa veguide is insignificant. production.

The studies carried out show that the developed filter should be technologically advanced during serial

\section{CONCLUSION}

Modern narrow-band compact filter, based on high-quality waveguide-dielectric resonator with anisotropic materials was designed. The designed filter satisfies contradictory requirements: it combines 
narrow bandwidth with low passband insertion loss. It is achieved by increasing the intrinsic quality factor of the resonators to values of 20,000 and more in the 3-centimeter wave range. Such a high $Q_{0}$-factor as compared with traditional waveguide-dielectric resonators (for which the Q-factor is 5,000) provides a 90 degree resonator turn relatively to the H-plane of waveguide and the use of monocrystal materials. A distinctive feature of the proposed filters is a high intrinsic quality factor, a rare spectrum of parasitic resonances (up to twice the operating frequency and higher), a high steepness of the slopes of the amplitudefrequency characteristic, and also high manufacturability in the 3-centimeter wave range.

The developed filter can be used: to solve the electromagnetic compatibility problems and compaction of information channels of satellite communication systems and television in the microwave range; in microwave generators with low noise level; in microwave receiver preselectors; and in measuring instrumentation.

\section{REFERENCES}

[1] R. J. Cameron, C. M. Kudsia, and R. R. Mansour, Microwave filters for communication systems: Fundamentals, design and applications. Hoboken, NJ, USA: John Wiley \& Sons, Inc., 2018.

[2] J. R. Sanchez, C. Bachiller, M. Julia, V. Nova, H. Esteban, and V. E. Boria, "Microwave Filter Based on Substrate Integrated Waveguide with Alternating Dielectric Line Sections," IEEE Microwave and Wireless Components Letters, vol. 28, no. 11, pp. 990-992, Nov. 2018, doi: 10.1109/LMWC.2018.2871644.

[3] J.-S. Hong and M. J. Lancaster, Microstrip Filters for RF/Microwave Applications. New York, USA: John Wiley \& Sons, Inc., 2001.

[4] Y. S. Makimoto M., Microwave Resonators and Filters For Wireless Communication, vol. 4. Berlin, Heidelberg: Springer Berlin Heidelberg, 2001.

[5] T. A. Sheikh, J. Borah, and S. Roy, "Bandwidth Improvement in BPF using Microstrip Couple lines Bandwidth Improvement in BPF using Microstrip Couple lines," 2014, no. December, doi: 10.13140/2.1.3254.6881.

[6] B. C. Liu et al., "An HTS 20-pole microstrip filter for GSM-1800 base stations," Journal of Superconductivity and Novel Magnetism, vol. 16, no. 5, pp. 819-822, 2003, doi: 10.1023/A:1026250817656.

[7] P. C. of S. P. Company, "Micran," Tomsk, Russia. 2018, [Online]. Available: https://sbis.ru/contragents/7017211757/701701001.

[8] J. M. Wu, T. Bai, W. Z. Lu, F. Liang, and B. Luo, "Novel compact interdigital bandpass filters with multilayer stepped impedance resonators/folded quarter-wavelength resonators," Key Engineering Materials, vol. 547, pp. 1924, Apr. 2013, doi: 10.4028/www.scientific.net/KEM.547.19.

[9] A. Zakharov and M. Ilchenko, "Trisection microstrip delay line filter with mixed cross-coupling," IEEE Microwave and Wireless Components Letters, vol. 27, no. 12, pp. 1083-1085, Dec. 2017, doi: 10.1109/LMWC.2017.2759724.

[10] H. Ghorbaninejad and M. Khalaj-Amirhosseini, "Compact bandpass filters utilizing dielectric filled waveguides," Progress In Electromagnetics Research B, vol. 7, pp. 105-115, 2008, doi: 10.2528/pierb08031101.

[11] A. G. Yushchenko and D. B. Mamedov, "Comparative Characteristics of Bandpass Filters Based on WDR and Microstrip Resonators," Electrical and Electronic Engineering, vol. 2, no. 2, pp. 7-10, Aug. 2012, doi: 10.5923/j.eee.20120202.02.

[12] A. Pons-Abenza et al., "Design and implementation of evanescent mode waveguide filters using dielectrics and additive manufacturing techniques," AEU - International Journal of Electronics and Communications, vol. 116, p. 153065, Mar. 2020, doi: 10.1016/j.aeue.2020.153065.

[13] I. C. Hunter and M. Y. Sandhu, "Monolithic integrated ceramic waveguide filters," in IEEE MTT-S International Microwave Symposium Digest, Jun. 2014, pp. 1-3, doi: 10.1109/MWSYM.2014.6848299.

[14] D. D. Tatachuk, Y. V Didenko, V. I. Molchanov, A. S. Franchuk, and Y. M. Poplavko, "Thin dielectric resonators in microwaves," in 2017 IEEE 1st Ukraine Conference on Electrical and Computer Engineering, UKRCON 2017 Proceedings, May 2017, pp. 45-50, doi: 10.1109/UKRCON.2017.8100517.

[15] C. Tomassoni, S. Bastioli, and R. V Snyder, "Compact mixed-mode filter based on TE101 cavity mode and TE01\$ $\delta \$$ dielectric mode," IEEE Transactions on Microwave Theory and Techniques, vol. 64, no. 12, pp. 44344443, Dec. 2016, doi: 10.1109/TMTT.2016.2623714.

[16] A. V Strizhachenko, "Diplexer on cylindrical waveguide with anisotropic crystal," Radioelectronics and Communications Systems, vol. 58, no. 6, pp. 269-273, Jun. 2015, doi: 10.3103/S0735272715060047.

[17] A. V Strizhachenko, S. V Pogorelov, S. N. Shulga, and A. A. Zvyagintsev, "Multi-section waveguide diplexer," Telecommunications and Radio Engineering (English translation of Elektrosvyaz and Radiotekhnika), vol. 78, no. 20, pp. 1817-1823, 2019, doi: 10.1615/TelecomRadEng.v78.i20.40.

[18] A. V Strizhachenko, "Approximate 3D model of electromagnetic modes in waveguide cross-shaped junctions with dielectric filling," Radioelectronics and Communications Systems, vol. 60, no. 12, pp. 562-568, Dec. 2017, doi: $10.3103 / \mathrm{S} 0735272717120068$.

[19] A. V Strizhachenko, "Electrodynamical model of composite cylindrical structure with laminated dielectric," Radioelectronics and Communications Systems, vol. 61, no. 1, pp. 38-43, Jan. 2018, doi: 10.3103/S0735272718010053.

[20] Y. Poplavko and Y. Yakymenko, Functional dielectrics for electronics: Fundamentals of conversion properties. Elsevier, 2020.

Int J Inf \& Commun Technol, Vol. 10, No. 3, December 2021: 198-203 


\section{BIOGRAPHIES OF AUTHORS}

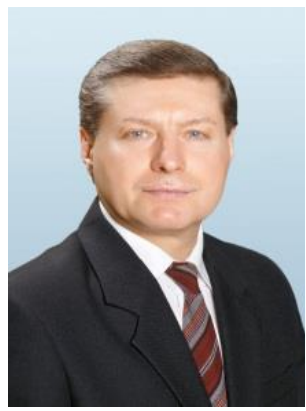

Strizhachenko Alexander Vladimirovich, Radiophysicist, Doctor of Physical and Mathematical Sciences (2014), Professor of the Department of Biophysics and Information Technologies, National University of Pharmacy, Kharkiv, Ukraine. The Area of Scientific Interest is: Electrodynamics of Complex Waveguide and Resonator Structures with Anisotropic Filling; Non-Destructive Measurements of the Parameters of anisotropic Materials in the Microwave Range; Design of Frequency-Separative Devices; Mathematical Modeling of Biological Objects.

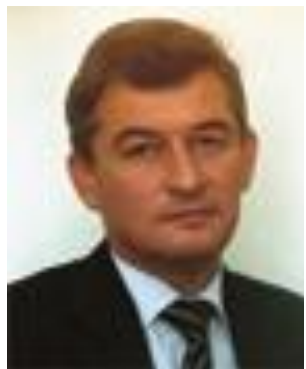

Shulga Sergey Nikolayevich, Radiophysicist, Doctor of Physical and Mathematical Sciences (2006), Professor, Dean of the School of Radiophysics, Biomedical Electronics and Computer Systems, V. N. Karazin Kharkiv National University, Kharkiv, Ukraine. The Area of Scientific Interests is: Ultra Wideband and Pulsed Antennas; Theoretical and Computational Methods for Analysis of the Complex-Composition Environments; Theoretical and Experimental Research of Metamaterials; Development of Medical Equipment for Magnetocardiography. 